\title{
DEFINIÇÃO DE HABILIDADES-ALVO PARA A CLASSIFICAÇÃO DE BRINCADEIRAS VOLTADAS ÀS CRIANÇAS COM TRANSTORNO DO ESPECTRO DO AUTISMO
}

\author{
Francisca Francisete de Sousa Nunes Queiroz'1, Christina César Praça Brasil', Fabiana Neiva \\ Veloso Brasileiro ${ }^{2}$, Felícia Gabler ${ }^{2}$, José Eurico de Vasconcelos Filho ${ }^{3}$ \\ 1,2 Universidade de Fortaleza, Brasil. zete.queiroz@hotmail.com; cpraca@unifor.br \\ fabianaveloso@unifor.br; feliciagabler@icloud.com \\ ${ }^{3} \mathrm{NATI}$ - Universidade de Fortaleza, Brasil. euricovasconcelos@unifor.br
}

\begin{abstract}
Resumo. $\mathrm{O}$ ato de brincar é uma estratégia que estimula o desenvolvimento e a aprendizagem de habilidades sob os aspectos cognitivos, físicos e sociais. A classificação de brincadeiras a partir de habilidades-alvo, com suas respectivas caracterizações e níveis, permite a estimulação do desenvolvimento global de crianças com Transtorno do Espectro do Autismo (TEA). Este estudo objetivou definir as habilidades-alvo para a classificação de brincadeiras a serem incorporadas no aplicativo Interautismo, fomentando um melhor direcionamento das atividades lúdicas para as crianças com Transtorno do Espectro do Autismo. O estudo respaldou-se em uma revisão de literatura, realizada de agosto a setembro de 2019, levando a alocação das brincadeiras em três eixos de habilidades-alvo: comunicativas, sociais e motoras. Verifica-se que a categorização das brincadeiras, de acordo com as habilidades-alvo definidas, oferece subsídios aos profissionais de saúde, cuidadores, pais e familiares, para que melhor direcionem o brincar das crianças com TEA, no sentido de contribuir para o seu desenvolvimento.
\end{abstract}

Palavras-chave: Transtorno do Espectro do Autismo; Habilidades para a Vida; Desenvolvimento Infantil; Brincadeiras; Aplicativos Móveis

\section{DEFINITION OF TARGET SKILLS FOR THE CLASSIFICATION OF GAMES DIRECTED TO CHILDREN WITH AUTISM SPECTRUM DISORDER}

\begin{abstract}
The act of playing is a strategy that encourages the development and learning of skills under the cognitive, physical and social aspects. The classification of games in target skills, with their respective characterizations and levels, allows the stimulation of the global development of children with Autism Spectrum Disorder (ASD). The objective of this study was to define the target skills for the classification of games to be incorporated into Interautism, promoting a better direction of play activities for children with Autism Spectrum Disorder. The study was supported by a literature review, held from august to september 2019, leading to the allocation of games in three axes of target skills: communicative, social and motor. It can be seen that the categorization of play, according to the target skills, offers subsidies to health professionals, caregivers, parents and family members, so that they can better direct the play of children with ASD, in order to contribute to their development.
\end{abstract}

Keywords: Autism Spectrum Disorder; Adaptation; Child Development; Play and Playthings; Mobile Applications

\section{INTRODUÇÃO}

O desenvolvimento infantil inicia-se desde a concepção, englobando o crescimento físico, a maturação neurológica, o desenvolvimento comportamental, sensorial, cognitivo e de linguagem, assim como as relações sociais e afetivas. Tem como efeito tornar a criança capaz de responder às suas necessidades e as do meio em que vive, considerando o seu 
contexto de vida, sendo um processo multidimensional e integral (Brasil, 2016; Organização Pan-Americana da Saúde, 2005). Quando se fala em desenvolvimento infantil, o ato de brincar é essencial, por ser uma das principais ações realizadas pelas crianças. (Godin, Freeman, \& Rigby, 2019).

O brincar conquista espaço e importância em todas as abordagens referentes à infância, principalmente como recurso para o desenvolvimento e a aprendizagem de habilidades (Cotonhoto, Rossetti, \& Missawa, 2019). Brincar possibilita à criança o aprimoramento da comunicação, da expressão, do relacionamento e da aprendizagem; uma vez que permite a experimentação de ações sensoriomotoras, a manipulação de objetos, o estabelecimento de interações sociais e a construção de representações mentais (Lucisano, Novaes, Sposito, \& Pfeifer, 2017). Cipriano e Almeida, (2016) apontam que o ato de brincar também evidencia possíveis deficits e dificuldades apresentadas por crianças com Transtorno do Espectro do Autismo (TEA), possibilitando identificar e acompanhar algumas alterações no desenvolvimento, uma vez que, ao brincar, as crianças revelam suas habilidades físicas, cognitivas e sociais.

O Transtorno do Espectro do Autismo (TEA), hoje classificado pelo Diagnostical and Statistical Manual of Mental Dosorders (DSM-V), configura-se como uma alteração global do desenvolvimento com prejuízos na comunicação social, além de comportamentos restritos e repetitivos. Isto é observado pelo fato de muitos dos seus portadores apresentarem problemas no desenvolvimento social e cognitivo (Apa, 2014).

O Center for Disease Control and Prevention (CDC) publicou um relatório que mostra o aumento da prevalência de casos em até sete vezes nos Estados Unidos, apresentando uma estimativa de 1 para 59 crianças (Baio et al., 2018). No Brasil, suspeita-se que $1 \%$ da população brasileira possua diagnóstico de autismo (Rocha, Souza, Costa \& Portes, 2019). Nesse sentido, intervenções especializadas em tempo hábil podem prever o prognóstico e a evolução do tratamento, reduzindo limitações e prejuízos ao desenvolvimento dessas crianças (Rossi, Lovisi, Abelha, \& Gomide, 2018).

Tendo em vista que a criança com TEA apresenta dificuldades em diversas áreas do desenvolvimento (interação social, comunicação, dentre outros), pode também apresentar comportamentos que prejudicam a experiência do brincar, visto que se limitam, na maioria das vezes, a um brincar isolado e disfuncional, não permitindo a interferência e a participação de outras pessoas (Calegari, Luz, Campos, Borba \& Ribeiro, 2018). 
Nesse contexto, instrumentos que se fundamentam na utilização do brincar como recurso de auxílio ao processo terapêutico, com foco nas habilidades necessárias ao desenvolvimento integral, são considerados importantes para profissionais que trabalham com crianças e para o aumento do envolvimento dos pais e/ou familiares com seus filhos. Esses instrumentos podem monitorar os progressos obtidos ao longo do tratamento e auxiliar nas decisões acerca da intervenção mais apropriada para cada caso (Cipriano \& Almeida, 2016).

A expansão dos recursos tecnológicos tem facilitado a vida das pessoas e ajudado os profissionais de saúde a promoverem o cuidado mediado por novos meios, a exemplo das tecnologias móveis (e.g. smartphones e tablets), caracterizando a área de saúde móvel ( $m$ Health). Este é um ramo da saúde eletrônica (eHealth) definido como a utilização de recursos tecnológicos de computação e comunicações móveis para a realização de cuidados e promoção de saúde (Free, Phillips, Felix, Galli, Patel, \& Edwards, 2010).

As tecnologias mobile constituem elementos de mediação e prescrição de atividades entre profissionais de saúde e pacientes, transpondo barreiras físicas e temporais, estendendo e ampliando o cuidado e a atuação dos profissionais de saúde, com informações, monitoramento, lembretes, jogos e promoção de atividades de autocuidado em saúde, sendo, nesse contexto, também adotadas a favor de crianças com TEA para o desenvolvimento de várias habilidades.

A proposta do brincar inserido nas tecnologias móveis surge como um apoio e contribui como ferramenta coadjuvante para o desenvolvimento integral de crianças com TEA, ampliam o repertório de diferentes profissionais da educação, da saúde e de seus familiares, ao estimularem essa população (Aragão, Junior \& Zaquel, 2019).

O projeto Interautismo (Bastos, Macambira, Carvalho, Vasconcelos Filho, Vasconcelos, \& Maia, 2018) segue a linha da mHealth, tendo como produto um sistema de apoio ao desenvolvimento da criança com TEA. O sistema contempla um aplicativo para dispositivos móveis voltado à família da criança com TEA e um sistema web de prescrição de atividades lúdicas (brincadeiras) para uso dos profissionais de saúde. A ideia é que o(s) profissional(is) possam prescrever brincadeiras a serem realizadas em âmbito domiciliar e acompanhadas via aplicativo.

Percebeu-se, entretanto, em avaliações da versão 1.0 da ferramenta que os profissionais de saúde, para uma prescrição adequada, precisavam de um bom conhecimento 
multidisciplinar sobre as atividades lúdicas para atender as peculiaridades da criança. Nesse contexto, observou-se a necessidade de, ainda na etapa de prescrição (ferramenta web), oferecer suporte ao profissional na indicação das brincadeiras. Para isso, verificou-se serem requeridas a classificação das brincadeiras de acordo com as habilidades a serem estimuladas e a implementação de um sistema de recomendação.

Assim, este estudo justifica-se pela necessidade de fomentar um melhor direcionamento do brincar para as crianças com TEA, no sentido de desenvolver habilidades que podem estar prejudicadas, bem como pela necessidade de categorização das atividades lúdicas de acordo com as principais habilidades-alvo para a estimulação do desenvolvimento infantil.

Diante do exposto, objetivou-se definir as habilidades-alvo para a classificação de brincadeiras a serem incorporadas ao Interautismo, fomentando um melhor direcionamento das atividades lúdicas para as crianças com TEA.

\section{METODOLOGIA}

Realizou-se uma revisão bibliográfica em duas etapas, quais sejam. Na primeira etapa, realizou-se a formulação do problema com o levantamento bibliográfico, além da coleta das informações, fatos e dados contidos no material bibliográfico selecionado (Gil, 2010). Na segunda etapa, o material selecionado foi analisado.

A primeira etapa ocorreu de agosto a setembro de 2019, buscando artigos científicos, livros, teses e dissertações publicados na área de saúde e de educação. As produções foram selecionadas no recorte temporal de 2010 a 2019, nas principais bases de dados: Capes, EBSCOhost, BDTD_IBICT, Scielo (Scientific Electronic Library Online), Redalyc e Scholar (Google acadêmico), utilizando-se os descritores e seus correspondentes em inglês (DeCS), quais sejam: autismo (autistic disorder); habilidades (skills), brincadeiras e brinquedos (play and playthings), atividades lúdicas (play activities) e desenvolvimento infantil (child development).

A questão norteadora para proceder a busca na literatura foi a seguinte: Quais habilidades podem ser desenvolvidas por meio da utilização de atividades lúdicas para estimular o desenvolvimento global de crianças com Transtorno do Espectro Autista (TEA)?

Os critérios de inclusão definidos para a seleção dos artigos foram os seguintes: textos que abordam o brincar para o desenvolvimento infantil, conceitos, metodologias e ferramentas 
existentes sobre os programas de ensino e a estimulação de crianças com TEA; além de textos que contemplam a questão norteadora. Foram excluídos os textos que não apresentavam conteúdo relevante para a temática, os que incluíam outros grupos (idosos, adultos) e os que não se referiam as crianças com TEA.

Na segunda etapa, realizou-se a análise do material selecionado, a partir de reuniões com a equipe multidisciplinar do projeto para o aprofundamento da leitura, a partir de técnicas de leitura, principais estratégias de análise na pesquisa bibliográfica (Lima \& Mioto, 2007). As técnicas utilizadas foram: leitura de reconhecimento do material bibliográfico (localizar e selecionar o material que pode estar relacionado com o tema da pesquisa); leitura exploratória (verificação se as informações selecionadas interessam ao estudo); leitura seletiva (determinação do material que interessa, relacionando-o aos objetivos da pesquisa); leitura reflexiva ou crítica (estudo crítico do material selecionado, organizando e sumarizando as informações) e leitura interpretativa (relação e integração das ideias expressas nas obras com o problema para o qual se busca resposta).

Nessa etapa, foram selecionados 35 textos que se alinhavam ao objeto de estudo. Ressaltase que foi utilizada a abordagem qualitativa para identificação, síntese dos principais achados, mapeamento das brincadeiras já prescritas na literatura e discussão das temáticas, favorecendo a definição das habilidades-alvo.

Para melhor compreender as habilidade-alvo, os pesquisadores recorreram ao 'Verbal Behavior Milestones Assessment and Placement Program' (VB-MAPP) de autoria Mark L. Sundberg, que oferece uma avaliação sistematizada para crianças com TEA ou com atrasos similares e constitui um instrumento de uso frequente para aqueles que planejam e implementam atendimentos nessa área.

O VB-MAPP é um programa que se baseia no desenvolvimento efetivo de habilidades, bem como determina se estas estão presentes ou ausentes, verifica o nível operante dos comportamentos da criança, fornece direção para a intervenção, além de oferecer um sistema de rastreamento de aquisição das habilidades da criança (Sundberg, 2008). 


\section{RESULTADOS E DISCUSSÃO}

A amostra final desta revisão foi constituída por trinta cinco textos científicos, os quais são apresentados no Quadro 1:

Quadro 1 - Quantitativo de publicações associado à base de dados e ao tipo

\begin{tabular}{|l|c|c|}
\hline \multicolumn{1}{|c|}{ Base de Dados } & N & Tipo \\
\hline Scielo & 5 & Artigos \\
\hline Capes & 4 & Artigos \\
\hline EBSCOhost & 5 & Artigos \\
\hline Redalyc & 1 & Artigos \\
\hline SCHOLAR & 11 & Artigos \\
\hline BDTDibict & 3 & Dissertações e teses \\
\hline Livros & 6 & Livros \\
\hline \multicolumn{2}{r}{} \\
\hline
\end{tabular}

\subsection{Definição das habilidades-alvo para classificar as brincadeiras}

Conforme a análise dos dados, foram definidas as habilidades-alvo importantes para o desenvolvimento integral das crianças, as quais são apresentadas por categorização. A proposta não tem o intuito de separar as competências que são adquiridas ao longo do processo de desenvolvimento do indivíduo. A intenção é evidenciar as habilidades mais recrutadas em determinados contextos e, assim, conduzir o brincar com mais singularidade, atendendo às necessidades para o desenvolvimento das crianças com TEA. Diante do exposto, descrevem-se, a seguir, cada uma das habilidades eleitas como essenciais: comunicativas, sociais e motoras.

\subsubsection{Habilidades comunicativas}

É nos primeiros anos de vida, antes da primeira década, que ocorre a maturação das habilidades comunicativas (Goulart \& Chiari, 2012), com a aquisição das áreas motoras, cognitivas e o controle da linguagem, elementos fundamentais para o desenvolvimento global. Segundo Oliveira, Flores, \& Souza, (2012) cada indivíduo utiliza-se da comunicação verbal e não-verbal para conseguir atingir propósitos, compartilhar interesses, pensamentos e emoções.

No âmbito das habilidades comunicativas constantes neste estudo, a proposta das atividades lúdicas a serem desenvolvidas almeja o enfrentamento dos distúrbios do desenvolvimento infantil que atingem o comportamento verbal. Segundo a revisão de literatura realizada para respaldo desta habilidade, destaca-se o Comportamento Verbal, 
descrito pela primeira vez em 1957 por Burrhus Frederic Skinner, que estabeleceu bases para formação de uma linha de pesquisa independente sobre a aquisição de linguagem e o estudo do comportamento humano, principalmente em crianças com TEA e outros atrasos do desenvolvimento (Skinner, 1957).

O referido estudo promoveu a abordagem do comportamento verbal, a partir do modelo 'Applied Behavior Analysis' (ABA) (Vismara \& Rogers, 2010; Fernandes \& Amato, 2013) e do instrumento de avaliação 'Verbal Behavior Milestones Assessment and Placement Program' (VB-MAPP). O modelo ABA direciona-se ao uso da análise do comportamento verbal com ênfase na função das respostas verbais, possibilitando o ensino de competências desejadas por meio de um controle apropriado. Já o VB-MAPP constitui um protocolo de avaliação comportamental da linguagem para crianças com TEA e com atrasos semelhantes (Sundberg, 2008).

A característica central dessa habilidade proposta por Skinner é considerar que a análise da linguagem do comportamento verbal envolve todas as formas de comunicação: linguagem de sinais, troca de figuras, linguagem escrita, gestos ou qualquer outra forma que uma resposta de comunicação possa assumir, sendo que a base da análise é focalizada no indivíduo falante e no ouvinte (Dib, 2017).

O comportamento verbal é operante, visto que acontece quando o indivíduo se comporta e existe a mediação do outro.

Assim, o ouvinte responde aos estímulos verbais produzidos pelo falante. Isto é, nesse processo, o indivíduo é considerado falante ao comportamento verbal perante o outro e torna-se ouvinte, ao reagir aos estímulos verbais produzidos por outros indivíduos.

Portanto, a habilidade comunicativa acontece por meio dos operantes verbais, que são unidades de análise funcional do comportamento verbal compostas por uma resposta verbal (seja ela vocal, gestual, escrita, etc) e cada habilidade de linguagem (operante verbal), as quais são diferenciadas entre si pelos estímulos fornecidos pelo ambiente (Skinner, 1957).

Ressalta-se, que se expõem neste estudo alguns operantes verbais sugeridos por Skinner (1957), que constituem um repertório linguístico completo, classificado em dois tipos diferentes de comportamento: o falante e o ouvinte. Os operantes verbais descrevem as habilidades comunicativas apresentadas no quadro 2 : 
Quadro 2. Habilidades comunicativas descritas a partir de comportamentos e operantes verbais (adaptado do VB-MAPP)

\begin{tabular}{|c|c|c|}
\hline $\begin{array}{l}\text { Habilidades de } \\
\text { Comunicação }\end{array}$ & $\begin{array}{l}\text { Operantes } \\
\text { verbais }\end{array}$ & Descrição \\
\hline \multirow{4}{*}{ Do Falante } & Mando & $\begin{array}{l}\text { O falante realiza pedidos ou emite para remover itens ou } \\
\text { atividades indesejáveis }\end{array}$ \\
\hline & Tato & O falante identifica objetos, ações, eventos, etc. \\
\hline & Intraverbal & $\begin{array}{l}\text { O falante responde à pergunta dos outros ou conversa } \\
\text { quando as palavras são controladas por outras palavras. }\end{array}$ \\
\hline & Ecoico & $\begin{array}{l}\text { O falante repete os sons, palavras e frases que ouve; } \\
\text { imitação vocal }\end{array}$ \\
\hline \multirow{3}{*}{ Do Ouvinte } & $\begin{array}{l}\text { Seguir instruções } \\
\text { ou atender o } \\
\text { pedido de outros }\end{array}$ & $\begin{array}{l}\text { O ouvinte executa uma ação desejada, segue uma } \\
\text { instrução ou seleciona um item em uma serie com outros } \\
\text { itens }\end{array}$ \\
\hline & $\begin{array}{l}\text { Discriminação } \\
\text { Visual }\end{array}$ & Habilidade de percepção visual \\
\hline & $\begin{array}{l}\text { Emparelhamento } \\
\text { com o modelo } \\
\text { Matching-to- } \\
\text { sample }\end{array}$ & $\begin{array}{l}\text { Tarefas de discriminação visual como quebra-cabeça, } \\
\text { desenhos, padrões, por exemplo, discriminações que } \\
\text { exigem que a criança observe e discrimine por estímulos } \\
\text { visuais }\end{array}$ \\
\hline
\end{tabular}

Diante da revisão e análise detalhada da literatura, a caracterização das habilidades comunicativas partiu do embasamento teórico proposto por Skinner (1957) e citado por diversos estudos (Lovaas, 2003; Dib 2017; Martone, 2017). Nesse sentido, a criança com TEA pode apresentar atrasos no desenvolvimento do comportamento verbal, com déficits na aquisição de aprendizagem dos operantes verbais, os quais são considerados os mais cruciais por afetar outras áreas do desenvolvimento - educacional, cognitivo, social e o brincar (Dib, 2017). Ademais, estudos mostram que o brincar visa ensinar mais de um operante verbal (Martone, 2017), favorecendo a criação de oportunidades para a criança praticar aquilo que está faltando em seu repertório.

\subsubsection{Habilidades sociais}

A Habilidade Social diz respeito a capacidade das pessoas se comportarem de acordo com uma determinada situação, o que envolve comportamentos de aprendizagem que permitem ao indivíduo interagir efetivamente com outros, além de obter aceitação social (Badiah, 2018).

Nesse sentido, crianças com TEA, geralmente, mostram atrasos nas habilidades sociais, apresentando contato visual limitado, dificuldade em manter a interação com outras pessoas, dificuldade em entender e usar a comunicação não verbal (gestos e expressões faciais), bem como dificuldades em retribuir as conversas (Bohlander, Orlich, \& Varley, 2012). Ademais, observa-se que uma das funções mais prejudicadas nessas crianças está relacionada ao acesso do brincar simbólico, pois a brincadeira delas é marcada pela 
atividade repetitiva, pobreza ou inexistência de brincadeiras de imitação social, existindo um prejuízo significativo, especialmente, em brincadeiras como o faz-de-conta.

O brincar está presente na vida da criança de modo a permitir o desenvolvimento das habilidades sociais e contribuir para o aprofundamento de vínculos entre pais, filhos e familiares, favorecendo o desenvolvimento de competências afetivas e relacionais (I. A. L. Silva \& Silva, 2019).

Nesse sentido, avaliar esses comportamentos, a partir do ato de brincar, é considerado de extrema importância, pois oferece recursos para que os profissionais possam incrementar ou aprimorar suas práticas (Lucisano, Novaes, Sposito, \& Pfeifer, 2017). Assim, a proposta das Habilidades Sociais descrita neste estudo repercute sobre o desenvolvimento de componentes que envolvem estratégias de interação social.

Dentre os componentes que caracterizam essas habilidades sociais destacam-se: brincadeira social; atenção compartilhada; interação física; expressões e emoções (tomada de perspectiva); cumprimentos e conversação.

Para Martone (2017), a 'brincadeira social' abrange a interação com os outros (adultos e crianças), sendo que alguns comportamentos mais avançados incluem jogos de tabuleiros, brincadeira imaginária, dentre outros. É válido destacar que todas essas brincadeiras abrangem também o comportamento verbal, uma vez que possibilitam compartilhar ou trocar brinquedos, cooperar na brincadeira, terminar a brincadeira apropriadamente, arrumar os brinquedos, iniciar e manter a brincadeira.

Nesse processo, a 'atenção compartilhada' caracteriza-se pelo contato visual, atender e atentar às outras pessoas. A 'interação física', por sua vez, ocorre quando uma criança inicia a interação com outras crianças sem a interferência de um adulto, podendo essa interação ser verbal ou não verbal. As 'expressões e emoções' (tomada de perspectiva) evidenciamse nos atos de rotular e interpretar emoções pessoais e outras emoções, mostrando compaixão com o outro e adaptando o comportamento apropriadamente. Os 'cumprimentos' são comportamentos em que se atenta a outras pessoas, cumprimentando-as. Já a 'conversação' propõe-se a atentar e responder a linguagem, iniciar e sustentar uma conversa, ou seja, responder questões sociais como nome, idade, onde mora, dentre outras e fazer perguntas (Mckinnon \& Krempa, 2002). 


\subsubsection{Habilidades motoras}

O desenvolvimento motor consiste no aprimoramento de movimentos corporais ao longo do ciclo de vida, possibilitando a interação entre ambiente, homem e tarefas a serem realizadas no cotidiano. É um processo que se inicia no nascimento e cessa com a morte. Cada indivíduo tem um tempo peculiar para aprender e desenvolver suas habilidades motoras. Entretanto, o grau de domínio das habilidades motoras básicas está relacionado ao seu estilo de vida e nível de saúde (Silva, Andrade, Torres, \& Amorim, 2017).

Nesse sentido, é fundamental para o ser humano realizar tarefas do dia a dia; contudo, uma coordenação motora global efetiva implica em um maior desenvolvimento motor e controle dos movimentos, o que contribui com a qualidade de vida. Independentemente da idade, o indivíduo está propício e pronto a aprender movimentos novos e cada vez mais desenvolver a sua parte psicomotora, sendo possível alterar o nível de aquisição das habilidades motoras em qualquer fase da vida (Perfeito \& Souza, 2017).

Verli, Benassi, Gonçalves, \& Albino, (2017) afirmam que na infância ocorre a aquisição do repertório motor que servirá de base para as outras fases. É nesse período que a criança adquire o domínio de seu corpo em diversas posturas, aprende a se locomover pelo ambiente de diferentes formas e a manipular variados tipos de objetos. Nessa perspectiva, Soares \& Cavalcante Neto, (2015) indicam ser, especificamente, na infância que o ser humano começa o aprimoramento de suas habilidades. Em concordância com outros autores, pode-se entender que o desenvolvimento motor são as alterações progressivas do comportamento motor, no decorrer do ciclo da vida, realizadas pela interação entre as exigências da tarefa, a biologia do indivíduo e as condições do ambiente, ressaltando que fica cada vez mais evidente que os estímulos dados têm contribuição para o seu desenvolvimento (Soares \& Cavalcante Neto, 2015; Perfeito \& Souza, 2017).

Em outras palavras, o desenvolvimento motor na infância possibilita à criança o domínio do seu corpo em diferentes posturas, locomoção pelo ambiente, por meio do andar, do correr, do saltar e do manipular objetos e instrumentos diversos, ou seja, receber uma bola, arremessar, chutar e outros (Seize \& Borsa, 2017). Assim, o ato de explorar o movimento, a partir do brincar, é algo que se pode perceber no cotidiano. Portanto, essas vivências que as crianças experimentam brincando geram diversidade de movimento (Oliveira \& Strohschoen, 2019). 
Em crianças com TEA, o comportamento motor poderá apresentar prejuízos no que diz respeito ao planejamento e sequenciamento motor. Em concordância, o estudo de Cantarero, Lloyd, \& Celnik, (2013), cujo objetivo foi descrever e comparar a capacidade motora global visando as habilidades motoras grossas e finas de um grupo de crianças com TEA, constatou progressão dos déficits à medida que a idade avança.

Nesse aspecto, para essa habilidade, enfatizam-se as habilidades de imitação motora; coordenação motora fina e coordenação motora grossa. A imitação motora é um operante verbal e caracteriza-se em imitar as ações dos outros. Esse comportamento ajuda no desenvolvimento social e lúdico, assim como outras atividades realizadas em grupo. A coordenação motora fina requer atividades de pequenos músculos, como escrever, coordenar movimentos dos olhos e das mãos, criar peças de artes, etc. Já coordenação motora grossa recruta músculos maiores do corpo, como sentar, andar, correr e outros (Martone, 2017).

Assim, para que as habilidades motoras se desenvolvam de forma satisfatória, devem ser consideradas as características biológicas, as experiências de aprendizagem e os fatores sociais que influenciam diretamente no desenvolvimento e no comportamento. Dessa forma, o ato de brincar constitui uma ferramenta valiosa para a estimulação dessas habilidades (Soares \& Cavalcante Neto, 2015).

\section{CONCLUSÕES}

Os achados deste estudo sugerem uma perspectiva qualitativa que contribui para o incremento do brincar, fomentando o direcionamento da atividade lúdica para desenvolvimento infantil, uma vez que este é um fator importante para a sistematização de categorias que permitem a descrição e as especificações das habilidades-alvo para a incorporação de brincadeiras que poderão estimular o desenvolvimento integral da criança com TEA.

Outro fator a ser mencionado diz respeito a importância do mapeamento das brincadeiras, a partir das habilidades-alvo identificadas e descritas neste estudo, no intuito de que as crianças com TEA usuárias do Interautismo possam ter uma proposta diferenciada de atividades, de acordo com suas demandas e necessidades, o que foi possibilitado pelo olhar qualitativo da investigação. Desse modo, o estudo favorece a reflexão sobre a 
disponibilização de um repertório significativo de brincadeiras com maior resolução e ampliação de competências.

Diante dos resultados alcançados, a definição das habilidades-alvo possibilita a continuação do presente estudo, o que acontecerá com a alocação das brincadeiras mapeadas, a partir da literatura, nos eixos de cada uma das habilidades-alvo definidas (comunicativa, social e motora). Esse conjunto de dados serão implementados no aplicativo Interautismo com o objetivo de proporcionar um melhor direcionamento do brincar para as crianças com TEA, além de oferecer subsídios aos profissionais de saúde, cuidadores, pais e familiares. Acredita-se que insto poderá repercutir em um cuidado eficaz e em melhorias para a saúde e a qualidade de vida de todos os envolvidos.

Ademais, o estudo traz importantes contribuições para a pesquisa qualitativa, uma vez que proporciona reflexões sobre $o$ ato de brincar e sua influência no desenvolvimento de habilidades que são permeadas pelas subjetividades dos envolvidos.

\section{REFERÊNCIAS}

Aragão, M. C. M., Júnior, J. B. B., \& Zaqueu, L. C. C. (2019). O uso de aplicativos para auxiliar no desenvolvimento de crianças com transtorno do espectro autista. Olhares \& Trilhas, 21(1), 43-57.

Associação Americana de Psicologia (APA). (2014). Manual diagnóstico e estatístico de transtornos mentais: DSM-5, 5. ed. Porto Alegre-RS: Artmed.

Badiah, L. I. (2018). The importance of social skills for autism. Advances in Social Science, Education and Humanities Research, 272, 20-24.

Baio, J., Wiggins, L., Christensen, D. L., Maenner, M. J., Daniels, J., Warren, Z., ... \& Dowling, N. F. (2018). Prevalence of autism spectrum disorder among children aged 8 years - Autism and developmental disabilities monitoring network, 11 Sites, United States, 2014. Morbidity and mortality weekly report. Surveillance summaries, 67(6), 1-23.

Bastos, A. P. F., Macambira, S. M., Carvalho, R. F. M., Vasconcelos Filho, J. E., Vasconcelos, R. A. de \& Maia, J. A. (2018). Interautismo: aplicação mHealth de apoio ao tratamento de crianças com autismo. CBIS2018, Fortaleza.

Bohlander, A. J., Orlich, F. \& Varley, C. K. (2012). Social skills training for children with autism. Departement of Psychiatry, 59(1), 165-174.

Brasil. Ministério da Saúde. Secretaria de Atenção à Saúde. (2016). Diretrizes de estimulação precoce: crianças de zero a 3 anos com atraso no desenvolvimento neuropsicomotor. Ministério da Saúde, Secretaria de Atenção à Saúde. Brasília: Ministério da Saúde.

Calegari, T., Luz, J. H., Campos, Y. A. E. S., Borba, R. I. H. \& Ribeiro, C. A. (2018). A criança autista em sessão de brinquedo terapêutico dramático: uma revisão winnicottiana. Revista Sociedade Brasileira Enfermagem Pediátrica. 18(1), 43-48.

Cantarero, G., Lloyd, A., \& Celnik, P. (2013). Reversal of long-term potentiation-like plasticity processes after motor learning disrupts skill retention. Journal of Neuroscience, 33(31), 12862-12869. 
Cipriano, M. S. \& Almeida, M. T. P. (2016). O brincar como intervenção no transtorno do espectro do autismo. Extensão em Ação, 2(11), 78-91.

Cotonhoto, L. A., Rossetti, C. B., \& Missawa, D. D. A. (2019). A importância do jogo e da brincadeira na prática pedagógica. Revista Construção Psicopedagógica, 27(28), 37-47.

Dib, R.R. (2017). O ensino de mando vocal para crianças com Transtorno do Espectro Autista: o efeito do atraso gradualdo modelo ecoico. Dissertação de Mestrado. Mestrado em Psicologia Experimental: Analise do Comportamento. Pontifícia Universidade Católica de São Paulo, São Paulo, Brasil.

Fernandes, F. D. M., \& Amato, C. A. L. H. (2013). Análise de comportamento aplicada e distúrbios do espectro do autismo: revisão de literatura. CoDAS, 25(3), 289-96.

Free, C., Phillips, G., Felix, L., Galli, L., Patel, V., \& Edwards, P. (2010). The effectiveness of M-health technologies for improving health and health services: a systematic review protocol. BMC research notes, 3(250), 1-7.

Gil, A. C. (2010). Como elaborar projetos de pesquisa. 5a ed. São Paulo: Atlas.

Godin, J., Freeman, A. \& Rigby, P. (2019) Conceptual clarification of the playful engagement in social interaction of preschool-aged children with autism spectrum disorder (ASD), Early Child Development and Care, 189(3), 430-440.

Goulart, B. N. G. de, \& Chiari, B. M. (2012). Comunicação humana e saúde da criança: reflexão sobre promoção da saúde na infância e prevenção de distúrbios fonoaudiológicos. Revista CEFAC, 14(4), 691-696.

Lima, T. C. S. de, \& Mioto, R. C. T. (2007). Procedimentos metodológicos na construção do conhecimento científico: a pesquisa bibliográfica. Revista Katálysis, 10(spe), 37-45.

Lovaas, O. I. (2003). Teaching individuals with developmental delays: Basic intervention techniques. Austin,: ProEd.

Lucisano, R. V., Novaes, L. C., Sposito, A. M. P, \& Pfeifer, L. I. (2017). Avaliação do brincar de faz de conta de pré-escolares: Revisão integrativa da literatura. Revista Brasileira Educação Especial, 23(2), 309-322.

Martone, C. C. M. (2017). Tradução e adaptação do Verbal Behavior Milestones Assessment and Placement Program(VB-MAPP) para a língua portuguesa e a efetividade do treino de habilidades comportamentais para qualificar profissionais. Tese de doutorado. Programa de Pós-Graduação em Psicologia, Universidade Federal de São Carlos, São Carlos, SP, Brasil.

McKinnon, K., \& Krempa, J. (2002). Social Skills Solutions: A Hands-On manual for teaching social skills to children with autism. Drl Books, Inc.

Oliveira, L. D., Flores, M. R., \& Souza, A. P. R. de. (2012). Fatores de risco psíquico ao desenvolvimento infantil: implicações para a fonoaudiologia. Revista CEFAC, 14(2), 333-342.

Oliveira, A. M., \& Strohschoen, A. P. G. A. (2019). A importância da ludicidade para inclusão do aluno com transtorno do espectro autista (TEA). Revista Eletrônica Pesquiseduca, 11(23), 127-139.

Organização Pan-Americana da Saúde (2005). Manual para vigilância do desenvolvimento infantil no contexto da AIDPI. Washington, D.C.

Perfeito, R. S., \& Souza, L. M. V. (2017). Relação entre os níveis de habilidade motora e a flexibilidade em escolares de 11 a 12 anos do Estado do Rio de Janeiro: um estudo descritivo de corte transversal. Revista Brasileira de Prescrição e Fisiologia do Exercício, 11(64), 44-54.

Rossi, L. P., Lovisi, G. M., Abelha, L, \& Gomide, M. (2018). Caminhos virtuais e autismo: acesso aos serviços de saúde na perspectiva da análise de redes sociais. Ciência \& Saúde Coletiva, 23(10), 1-8. 
Rocha, C. C., Souza, S. M. V., Costa, A. F., \& Portes, J. R. M. (2019). O perfil da população infantil com suspeita de diagnóstico de transtorno do espectro autista atendida por um Centro Especializado em Reabilitação de uma cidade do Sul do Brasil. Physis: Revista de Saúde Coletiva, 29(4), 1-20.

Seize, M. de M.; \& Borsa, J. C. (2017). Instrumentos para rastreamento de sinais precoces do autismo: revisão sistemática. Psico-USF, 22(1), 161-176.

Silva, M, F. dos S., Andrade, A. P., Torres M, F. de P., \& Amorim, G. C.C. (2017). As brincadeiras das crianças de ontem e de hoje no contexto sociocultural, HOLOS, Ano 33, 03, 1-13.

Silva, I. A. L., \& Silva, M. F. G. (2019). A importância da brincadeira de faz de conta na educação infantil: sob o olhar de professoras. Revista Zero-a-seis. 21(39), 67-80.

Skinner, B. F. (1957). Verbal behavior. New York: Appleton-Century-Crofts

Soares, A. M., \& Cavalcante Neto, J. L. (2015). Avaliação do comportamento motor em crianças com transtorno do espectro do autismo: Uma revisão sistemática. Revista Brasileira de Educação Especial, 21(3), 445458.

Sundberg, M. L. (2008). The verbal behavior milestones assessment and placement program: The VB-MAPP. Concord, CA: AVB Press.

Verli, M. V. de A., Benassi, R., Gonçalves, L. C. O., \& Albino, F. (2017). Comparação do nível da equilibrarão entre crianças praticantes de atividade física regular e crianças que não praticam atividades físicas no ensino fundamental. Revista Brasileira de Prescrição e Fisiologia do Exercício, 11(66), 315-328.

Vismara, L. A., Rogers, S. (2010). Behavioral treatments in autism spectrum disorder: what do we know? Annual Review of Clinical Psychology, 6, 447-68. 